\title{
ON MONODROMIES OF A DEGENERATION OF IRREDUCIBLE SYMPLECTIC KÄHLER MANIFOLDS
}

\author{
YASUNARI NAGAI
}

\begin{abstract}
We study the monodromy operators on the betti cohomologies associated to a good degeneration of irreducible symplectic manifold and we show that the unipotency of the monodromy operator on the middle cohomology is at least the half of the dimension. This implies that the "mildest" singular fiber of a good degeneration with non-trivial monodromy of irreducible symplectic manifolds is quite different from the generic degeneration of abelian varieties or Calabi-Yau manifolds.
\end{abstract}

\section{INTRODUCTION}

For the study of smooth algebraic (or analytic) varieties, it is often important and useful to consider its degenerations. One can see this principle, for example, by remembering the role played by the singular fibers in the theory of elliptic surfaces due to Kodaira.

The Kodaira singular fibers are completely described in terms of the periods and monodromies around the singular fiber. The study of the periods and degenerations of abelian varieties is one of the most direct generalizations of the theory of elliptic surfaces to higher dimensions. The theory of periods is generalized by Griffiths and many other contributers to the general situation using the variation of Hodge structures. Since then, many significant results are proved using this mechanism. In some cases, the Hodge structure on the middle cohomology, i.e. $H^{n}(X, \mathbb{C})$ for $X$ of dimension $n$, plays an important role. As an example, we can recall the classification of Kulikov models for degeneration of K3 surfaces $[\mathrm{Ku}, \mathrm{P}-\mathrm{P}]$ and the proof of global Torelli theorem for K3 surfaces via Kulikov models by Friedman $[\mathrm{Fr}]$.

An irreducible symplectic Kähler manifold is a generalization of a K3 surface to higher dimension. As is well known, the local Torelli theorem holds for irreducible symplectic manifolds on the second cohomology group (not only on the middle cohomology). From this result, One can easily suppose some strong similarities between the irreducible symplectic manifolds and $\mathrm{K} 3$ surfaces. There is no reason that prevents us from studying degenerations of irreducible symplectic manifolds.

Date: Preliminary Version: 2006.05.08.

2000 Mathematics Subject Classification. Primary 14D05, Secondary 14J32, 32Q20. 
But if we consider the period and monodromy only on the second cohomology as invariants of the degeneration of irreducible symplectic manifolds, it seems that one misses some important informations. Since the weight of the Hodge structure on the second cohomology is 2, the index of unipotency of the monodromy is either 0,1 , or 2 . The monodromy operator should have some information about the "complexity" of the degenerate fiber. In the case of Kulikov models of K3 surfaces or toroidal degenerations of abelian varieties, the unipotency of the monodromy on the middle cohomology certainly corresponds to the combinatorial complexity of the singular fiber. There are $(n+1)$ patterns of local combinations of the components of the degenerate variety with normal crossings of dimension $n$, so the unipotency of the monodromy on the second cohomology, a priori, may not fully reflect the combinatorial information of the degenerate fiber.

In this article, we examine the relation between the monodromy operators on the cohomologies, in particular the relation between the monodromies on the second cohomology and the middle cohomology.

To consider this problem in a general situation, we assume a semi-stable model which is called a good degeneration (see Definition 4.2). In fact, we can construct an example of a good degeneration (Theorem 4.3). Our main result is the following.

Theorem (Theorem 5.3). Let $\pi: \mathscr{X} \rightarrow \Delta$ be a good degeneration of irreducible symplectic $2 n$-folds. Let $T_{2 n}$ be the monodromy operator on $H^{2 n}\left(\mathscr{X}_{t}, \mathbb{C}\right)(t \neq 0)$ associated to the family $\pi$, and $N_{2 n}=\log T_{2 n}$. Assume $N_{2 n}^{n}=0$, then $N_{2 n}=0$.

The maximal $l$ with $N_{2 n}^{l} \neq 0$ is called the unipotency of $T_{2 n}$. This theorem asserts that the unipotency of the monodromy on the middle cohomology associated to a good degeneration of irreducible symplectic manifold is 0 or not less than $n$. This is quite different from the situation one can expect in the case of general semi-stable degeneration. For example, we can easily show the following corollary.

Corollary (See Corollary 5.5). Consider a good degeneration of irreducible symplectic 2 -folds with non-trivial monodromy on the middle cohomology, and let $\Gamma$ be the dual graph of the configuration of the irreducible components of the singular fiber $X$. Then the dimension of the topological realization $|\Gamma|$ is at least $n$.

This phenomenon can be seen as an aspect of the general principle that the geometry of irreducible symplectic manifold is very restrictive.

Plan of the article. This article consists of five sections: In the first section, we review some necessary definitions about symplectic Kähler manifolds, degenerations and monodromy associated to them. In $\S 2$, we consider an example of degeneration of irreducible symplectic manifold arising from a family of K3 
surfaces by the Hilbert scheme construction and compute the monodromies associated to it. In fact, this example motivates this research. The next section is devoted to the study of the family of generalized Kummer varieties and its associated monodromy. In $\S 4$, we define a notion of a good degeneration of symplectic Kähler manifold. In the last section, we state a conjecture based on the examples in $\S \S 2,3$, and get a partial answer to the conjecture for good degenerations of irreducible symplectic manifold.

Acknowledgemenet. The author is financially supported by PD Fellowship of Korea Institute for Advanced Study (KIAS).

\section{DEFINITIONS AND NOTATIONS}

In this section, we collect some basic definitions and notations which are needed in this article.

Symplectic Kähler manifold. Let us start with the review of some basics of compact symplectic Kähler manifold. A fundamental reference is [Be]. See also [Huy1, Huy2].

Definition 1.1. Let $X$ be a compact Kähler manifold of dimension $2 n$. A holomorphic 2-form $\sigma \in H^{0}\left(X, \Omega_{X}^{2}\right)$ on $X$ is a (holomorphic) symplectic form if its top exterior power $\sigma^{\wedge n} \in H^{0}\left(X, \Omega_{X}^{2 n}\right)$ is nowhere vanishing. The pair $\left(X, \sigma_{X}\right)$ is called a holomorphic symplectic Kähler manifold. $X$ is said to be an irreducible symplectic if the following conditions are satisfied.

(i) There exists a holomorphic symplectic form $\sigma$.

(ii) The space of global holomorphic 2-forms $H^{0}\left(X, \Omega_{X}^{2}\right)$ is spanned by the symplectic form $\sigma$.

(iii) $X$ is simply connected.

Given an irreducible symplectic manifold $X$, we have a non-degenerate primitive quadratic form $q_{X}$ on $H^{2}(X, \mathbb{Z})$, which is called the Beauville-Bogomolov form.

Famous decomposition theorem for compact Kähler manifolds with trivial canonical bundle implies that a compact symplectic Kähler manifold is an étale quotient of a product of a complex torus and irreducible symplectic manifolds. There are few known examples of irreducible symplectic Kähler manifold. Here we describe two families of examples, which are classically known.

Example 1.2 ([BB] $]$ ). Let $S$ be a K3 surface and $\operatorname{Hilb}^{n}(S)$ the Hilbert scheme (or Douady space) of 0-dimensional sub-schemes of length $n$ on $S$. Then, $\operatorname{Hilb}^{n}(S)$ is an irreducible symplectic Kähler manifold of dimension $2 n$. For the second betti cohomology of $\operatorname{Hilb}^{n}(S)$, we have an isomorphism

$$
H^{2}\left(\operatorname{Hilb}^{n}(S), \mathbb{C}\right) \cong H^{2}(S, \mathbb{C}) \oplus \mathbb{C} \cdot[E],
$$


where $E$ is an irreducible exceptional divisor of the birational morphism

$$
\operatorname{Hilb}^{n}(S) \rightarrow \operatorname{Sym}^{n}(S),
$$

which is called the Hilbert-Chow morphism ([B] $]$ ) and decomposition in (1) is orthogonal with respect to the Beauville-Bogomolov form.

More generally, a compact connected component $M$ of the moduli space of stable sheaves on a K3 surface $S$ is known to be an irreducible symplectic manifold which is deformation equivalent to $\operatorname{Hilb}^{n}(S)$ of appropriate dimension. An irreducible symplectic manifold $X$ is said to be of Hilbert type if $X$ is deformation equivalent to some $\operatorname{Hilb}^{n}(S)$.

Example $1.3\left([\overline{\mathrm{Be}} \mid)\right.$. Let $A$ be a complex torus and consider $\operatorname{Hilb}^{n+1}(A)$ and its Albanese morphism $\alpha: \operatorname{Hilb}^{n+1}(A) \rightarrow A$, which is a locally trivial fiber bundle. Take its fiber $\operatorname{Kum}^{n}(A)=\alpha^{-1}(0)$. Then this is an irreducible symplectic Kähler manifold of dimension $2 n$. We can $\operatorname{regard} \operatorname{Kum}^{n}(A)$ as a resolution of $A^{n} / \mathfrak{S}_{n+1}$. Let $E$ be the exceptional divisor. Then $E$ is irreducible and we have

$$
H^{2}\left(\operatorname{Kum}^{n}(A), \mathbb{C}\right) \cong H^{2}(A, \mathbb{C}) \oplus \mathbb{C} \cdot[E]
$$

and this is also orthogonal with respect to the Beauville-Bogomolov form.

More recently, O'Grady constructed two sporadic examples of irreducible symplectic manifold [OG1, OG2]. It is noteworthy that these four types of examples are all of the known examples for the moment.

\section{Degeneration and Monodromy.}

Definition 1.4. Let $Y$ be a compact Kähler manifold and $\Delta$ a unit disk in $\mathbb{C}$. A flat proper morphism $\pi: \mathscr{X} \rightarrow \Delta$ of a normal complex Kähler space $\mathscr{X}$ is said to be a degeneration or degenerating family of $Y$ if $\pi$ is smooth over $\Delta-\{0\}$ and $\mathscr{X}_{t}=\pi^{-1}(t) \cong Y$ for some $t \in \Delta$. The fiber $X=\mathscr{X}_{0}=\pi^{-1}(0)$ is said to be the singular fiber if $\pi$ is not smooth.

Given a degenerating family $\mathscr{X}$, we have the monodromy operators on the cohomologies of $\mathscr{X}_{t}(t \neq 0)$.

Definition 1.5. Let $\pi: \mathscr{X} \rightarrow \Delta$ be a degenerating family. Then parallel displacement on the local system $R^{m}\left(\pi_{0}\right)_{*} \mathbb{C}$ on $\Delta-\{0\}$ induces a homomorphism

$$
T_{m}: \pi_{1}(\Delta-\{0\}) \rightarrow G L\left(H^{m}\left(\mathscr{X}_{t}, \mathbb{C}\right)\right),
$$

the monodromy representation. We denote also by $T_{m}$ the image of a generator under the monodromy representation, which is called the monodromy operator.

By the monodromy theorem, $T_{m}$ is quasi-unipotent, i.e. $\left(T_{m}^{k}-I\right)^{N}=0$ for some $k, N \in \mathbb{N}$. Therefore, changing the base by a cyclic cover $\Delta \rightarrow \Delta, t \mapsto t^{k}$, we can always make the monodromy operator unipotent. 
Definition 1.6. Let $T$ be an unipotent automorphism of a finite dimensional vector space over a field of characteristic zero. The logarithm of $T$ is defined by

$$
N=\log T=(T-I)-(T-I)^{2}+\cdots+(-1)^{n+1}(T-I)^{n}+\cdots .
$$

Note that the right hand side is a finite sum because $T$ is unipotent and the logarithm $N$ is a nilpotent endomorphism. Of course, we can reconstruct $T$ from $N$ by the exponential:

$$
T=\exp N=I+\frac{N}{1 !}+\frac{N^{2}}{2 !}+\cdots+\frac{N^{n}}{n !}+\cdots .
$$

We define the index of nilpotency of $N$ by

$$
\operatorname{nilp}(N)=\max \left\{k \mid N^{k} \neq 0\right\} .
$$

We mean by the index of unipotency of a unipotent $T$ the index of nilpotency of $N=\log T$.

If $T_{m}$ is the unipotent monodromy operator on $H^{m}\left(\mathscr{X}_{t}, \mathbb{C}\right)$ associated to a degeneration $\pi: \mathscr{X} \rightarrow \Delta$, then by the theory of variation of Hodge structures, more precisely, by $S L_{2}$-orbit theorem [Sc] for example, we know that the index of unipotency of $T_{m}$ is at most $m$, i.e.

$$
0 \leq \operatorname{nilp}\left(N_{m}\right) \leq m
$$

for $N_{m}=\log T_{m}$ (See also a lecture note by Griffiths [Topics], Chapter IV).

\section{EXAMPLE: THE CASE OF HilbeRT TYPE}

In this section, we consider degenerations of irreducible symplectic manifolds of Hilbert type, i.e. irreducible symplectic manifolds which is deformation equivalent to $\operatorname{Hilb}^{n}(S)$ for some K3 surface $S$.

Let us consider an easy example.

Example 2.1. Let $p: \mathscr{S} \rightarrow \Delta$ be a degeneration of $\mathrm{K} 3$ surfaces. Consider the Hilbert scheme $\overline{\mathscr{X}}_{n}=\operatorname{Hilb}^{n}(\mathscr{S} / \Delta)$ of 0-dimensional sub-schemes relative to $p$, and take the normalization $\mathscr{X}_{n} \rightarrow \overline{\mathscr{X}}_{n}$. Then, the natural morphism $\pi_{n}: \mathscr{X}_{n} \rightarrow \Delta$ is a degeneration of irreducible symplectic manifolds, whose general fiber $\left(\mathscr{X}_{n}\right)_{t}$ is isomorphic to $\operatorname{Hilb}^{n}\left(\mathscr{S}_{t}\right)$.

Consider the monodromy operator on the cohomologies associated to this degeneration. Let us assume that the monodromy operator $T^{\prime}$ on the second cohomology group $H^{2}\left(\mathscr{S}_{t}, \mathbb{C}\right)(t \neq 0)$ associated to the family $p: \mathscr{S} \rightarrow \Delta$ is unipotent and let $N^{\prime}=\log T^{\prime}$. Then by (2), we have

$$
\operatorname{nilp}\left(N^{\prime}\right)=0,1 \text {, or } 2 \text {. }
$$

Since we have $H^{2}\left(\operatorname{Hilb}^{n}\left(\mathscr{S}_{t}\right), \mathbb{C}\right)=H^{2}\left(\mathscr{S}_{t}, \mathbb{C}\right) \oplus \mathbb{C} \cdot\left[E_{t}\right]$ on the general fiber and the class $\left[E_{t}\right]$ is clearly invariant under the action of the monodromy $T_{2}$ on the 
second cohomology $H^{2}\left(\operatorname{Hilb}^{n}\left(\mathscr{S}_{t}\right), \mathbb{C}\right)$, we have

$$
T_{2}=T^{\prime} \oplus \mathrm{id}
$$

in particular, $T_{2}$ is unipotent. In fact we can say more about the monodromy operator $T_{m}$ on $H^{m}\left(\left(\mathscr{X}_{n}\right)_{t}, \mathbb{C}\right)$ :

Proposition 2.2. Notation as above. Then for $m \leqslant 2 n$,

(i) $T_{m}$ is unipotent.

(ii) Let $N_{2 k}=\log T_{2 k}$. Then, $\operatorname{nilp}\left(N_{2 k}\right)=k \cdot \operatorname{nilp}\left(N_{2}\right)$ for $k \leqslant n$. In particular $\operatorname{nilp}\left(N_{2 k}\right) \in\{0, k, 2 k\}$.

Proof. $\operatorname{Hilb}^{n}(S)$ is given as a resolution of the symmetric product $\operatorname{Sym}^{n}(S)$ and $E^{n} \neq 0$ for its irreducible exceptional divisor $E$. This implies that $H^{2 k}\left(\operatorname{Hilb}^{n}(S)\right)$ is the image of $\operatorname{Sym}^{k} H^{2}\left(\operatorname{Hilb}^{n}(S)\right)$ and $H^{2 k+1}\left(\operatorname{Hilb}^{n}(S)\right)=0$. In other words, $H^{*}\left(\operatorname{Hilb}^{n}(S)\right)$ is generated by $H^{2}\left(\operatorname{Hilb}^{n}(S)\right)$ as an algebra over $H^{0}\left(\operatorname{Hilb}^{n}(S)\right)=$ $\mathbb{C}$. In general we have the following proposition:

Proposition 2.3 (Verbitsky, [Bo]. See also [Huy2] §24). Let X be an irreducible symplectic manifold of dimension $2 n$ and let $S^{*}(X, \mathbb{C})$ be the sub-algebra of $H^{*}(X, \mathbb{C})$ generated by $H^{2}(X, \mathbb{C})$. Then

$$
S H^{*}(X, \mathbb{C}) \cong \operatorname{Sym}^{*} H^{2}(X, \mathbb{C}) /\left\langle\alpha^{n+1} \mid q_{X}(\alpha)=0\right\rangle
$$

where $q_{X}$ is the Beauville-Bogomolov form (Definition 1.1).

From this proposition, we know that $H^{2 k}\left(\operatorname{Hilb}^{n}(S)\right) \cong \operatorname{Sym}^{k} H^{2}\left(\operatorname{Hilb}^{n}(S)\right)$ for $k \leqslant n$. Therefore, $T_{2 k}=\operatorname{Sym}^{k} T_{2}$. The claims in the proposition immediately follows from the following lemma on linear algebra. Q.E.D.

Lemma 2.4. Let $V_{1}, V_{2}$ be finite dimensional vector spaces over a field of characteristic zero, $T_{i}$ be a unipotent automorphism on $V_{i}$, and $N_{i}=\log T_{i}$. Then $T_{1} \otimes T_{2}$ is also unipotent and

$$
\log \left(T_{1} \otimes T_{2}\right)=N_{1} \otimes I+I \otimes N_{2}
$$

on $V_{1} \otimes V_{2}$. Moreover, we have

$$
\operatorname{nilp}\left(\log \left(T_{1} \otimes T_{2}\right)\right)=\operatorname{nilp}\left(N_{1}\right)+\operatorname{nilp}\left(N_{2}\right) .
$$

In particular, $\mathrm{Sym}^{k} T_{1}$ on $\mathrm{Sym}^{k} V_{1}$ is unipotent and

$$
\operatorname{nilp}\left(\log \left(\operatorname{Sym}^{k} T_{1}\right)\right)=k \cdot \operatorname{nilp}\left(N_{1}\right) .
$$

Proof. The first assertion is just a property of exponentials and logarithms of the matrices. The second equality follows from

$$
\left(N_{1} \otimes I+I \otimes N_{2}\right)^{k}\left(v_{1} \otimes v_{2}\right)=\sum_{i=0}^{k}\left(\begin{array}{l}
k \\
i
\end{array}\right) N_{1}^{k}\left(v_{1}\right) \otimes N_{2}^{k-i}\left(v_{2}\right) .
$$

Since $\operatorname{Sym}^{k} V_{1} \subset V_{1}^{\otimes k}$, we have

$$
\operatorname{nilp}\left(\log \left(\operatorname{Sym}^{k} T_{1}\right)\right) \leqslant \operatorname{nilp}\left(\log \left(T_{1}^{\otimes k}\right)\right)=k \cdot \operatorname{nilp}\left(N_{1}\right)
$$


On the other hand, there exists $v_{1} \in V_{1}$ such that $N^{l}\left(v_{1}\right) \neq 0, N^{l+1}\left(v_{1}\right)=0$ for $l=\operatorname{nilp}\left(N_{1}\right)$. Therefore

$$
\left(\log \left(\operatorname{Sym} T_{1}\right)\right)^{k l}\left(v_{1}^{k}\right)=\left(N^{l}\left(v_{1}\right)\right)^{k} \neq 0,
$$

which shows the last claim.

Q.E.D.

Note that the proof of Proposition 2.2 does not use the fact that the degeneration of $\operatorname{Hilb}^{2 n}\left(\mathscr{S}_{t}\right)$ is induced from a degeneration of K3 surfaces as in Example 2.1. Therefore one can easily generalize Proposition 2.2 to the following theorem by the same proof.

Theorem 2.5. Let $\pi: \mathscr{X} \rightarrow \Delta$ is a degenerating family of irreducible symplectic manifolds whose general fiber is of Hilbert type of dimension $2 n$. Let $T_{m}$ be the monodromy operator associated to this family on $H^{m}\left(\mathscr{X}_{t}, \mathbb{C}\right)$ and assume that $T_{2}$ is unipotent. Then for $k \leqslant n, T_{2 k}$ is unipotent and $\operatorname{nilp}\left(N_{2 k}\right)=k \cdot \operatorname{nilp}\left(N_{2}\right)$, where $N_{2 k}=\log T_{2 k}$. In particular nilp $\left(N_{2 k}\right) \in\{0, k, 2 k\}$.

Remark 2.6. This theorem, more precisely, the equality

$$
H^{2 k}(X, \mathbb{C})=\operatorname{Sym}^{k} H^{2}(X, \mathbb{C})(k \leqslant n)
$$

for $X$ of Hilbert type means that all the periods on the cohomologies are governed only by the second cohomology. This can be interpreted as an evidence of the speculation that the period on the second cohomology strongly captures the moduli of irreducible symplectic manifolds of Hilbert type.

\section{EXAMPLE: A FAMILY OF GENERALIZED KuMMER VARIETIES}

From the results of Proposition 2.2 and Theorem 2.5, it is natural to ask whether there is some restriction on the index of unipotency of the monodromy operator for more general case. The life in general is not as simple as in the case of Hilbert type, since the cohomology ring of a general irreducible symplectic Kähler manifold is not generated by the second degree part, and even has non-zero odd degree, so some mysterious thing may happen in the higher degree cohomologies. To catch a glimpse of these general cases, we may use the family of generalized Kummer varieties as a test case.

Definition 3.1. Let $p: \mathscr{A} \rightarrow \Delta^{*}=\Delta-\{0\}$ be a smooth projective family of abelian surfaces with 0 -section and consider its relative Hilbert scheme of $(n+1)$ points $\tilde{\pi}: \operatorname{Hilb}^{n+1}\left(\mathscr{A} / \Delta^{*}\right) \rightarrow \Delta^{*}$. Then we have the commutative diagram

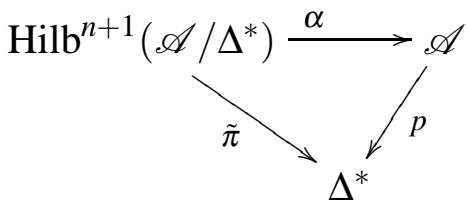

where $\alpha$ is the Albanese morphism over $\Delta^{*}$, and let $\operatorname{Kum}^{n}\left(\mathscr{A} / \Delta^{*}\right)$ be the inverse image of the 0 -section of $p$ by $\alpha$. Then $\pi: \operatorname{Kum}^{n}\left(\mathscr{A} / \Delta^{*}\right) \rightarrow \Delta^{*}$ is a family of generalized Kummer varieties of dimension $2 n$ (cf. Example 1.3). 
Remark 3.2. We should be able to compactify $\pi: \operatorname{Kum}^{n}\left(\mathscr{A} / \Delta^{*}\right) \rightarrow \Delta^{*}$ by allowing some mild singular fiber of $p$ over the origin as in the case of Hilbert schemes in $\S 2$. Or we can obtain some compactification using the embedding of $\pi$ to the projective space over $\Delta$ in an obvious way. But we do not discuss about the singular fiber here because we do not use any geometric information of the singular fiber in the sequel.

Let $p: \mathscr{A} \rightarrow \Delta^{*}$ be the family of abelian surfaces in Definition 3.1 Let $\bar{T}_{m}$ be the associated monodromy operator on $H^{m}\left(\mathscr{A}_{t}\right)$ and assume that $\bar{T}_{1}$ is unipotent. Then one can easily see that all $\bar{T}_{m}$ 's are unipotent and non-trivial. We denote $\bar{N}_{m}=\log \bar{T}_{m}$. Of course nilp $\left(\bar{N}_{1}\right) \leqslant 1$ and equality holds if $\bar{T}_{1}$ is non-trivial. Assume nilp $\left(\bar{N}_{1}\right)=1$ and let $l=\operatorname{rank} \bar{N}_{1}(=1,2)$. Then we know that

$$
\operatorname{nilp}\left(\bar{N}_{2}\right)=l, \quad \operatorname{nilp}\left(\bar{N}_{3}\right)=1, \quad \operatorname{nilp}\left(\bar{N}_{4}\right)=0 .
$$

Take the family of generalized Kummer $2 n$-folds $\pi: \operatorname{Kum}^{n}\left(\mathscr{A} / \Delta^{*}\right) \rightarrow \Delta^{*}$ and let $T_{m}$ be the monodromy operator on $H^{m}\left(\operatorname{Kum}^{n}\left(\mathscr{A}_{t}\right)\right)$ and $N_{m}=\log T_{m}$. As in $\S 2, N_{2}$ can be expressed by

$$
N_{2}=\bar{N}_{2} \oplus \mathrm{id}
$$

under $H^{2}\left(\operatorname{Kum}^{n}\left(\mathscr{A}_{t}\right), \mathbb{C}\right) \cong H^{2}(A, \mathbb{C}) \oplus \mathbb{C} \cdot\left[E_{t}\right]$. We calculate $\operatorname{nilp}\left(N_{2 k}\right)$ for $1<$ $k \leqslant n$ below.

We prepare a lemma.

Lemma 3.3. Let $\pi: \mathscr{X} \rightarrow \Delta$ be a degeneration of irreducible symplectic manifolds, and $T_{m}$ the associated monodromy operator on $H^{m}\left(\mathscr{X}_{t}, \mathbb{C}\right)$. Assume $T_{2}$ and $T_{2 k}$ are unipotent and take $N_{m}=\log T_{m}(m=2,2 k)$. Then, $\operatorname{nilp}\left(N_{2 k}\right) \geqslant$ $k \cdot \operatorname{nilp}\left(N_{2}\right)$. In particular $N_{2 k}^{l k}=0 \Rightarrow N_{2}^{l}=0$.

Proof. We again use the result of Verbitsky (Proposition 2.3). We have an injective homomorphism $S^{k}=\operatorname{Sym}^{k} H^{2}\left(\mathscr{X}_{t}\right) \hookrightarrow H^{2 k}\left(\mathscr{X}_{t}\right)$ and we have

$$
\operatorname{nilp}\left(N_{2 k}\right) \geqslant \operatorname{nilp}\left(N_{2 k \mid S^{k}}\right)=\operatorname{nilp}\left(\log \operatorname{Sym}^{k} T_{2}\right)=k \cdot \operatorname{nilp}\left(N_{2}\right)
$$

by Lemma 2.4

Q.E.D.

The main tool of our calculation is the following theorem:

Theorem 3.4 (Göttsche-Soergel [GS], Theorem 7). Let A be an abelian surface and fix a natural number $n$. Consider the set $P(n)$ of partitions of $n$, i.e.,

$$
P(n)=\left\{\alpha=\left(\alpha_{1}, \alpha_{2}, \cdots, \alpha_{n}\right) \mid \alpha_{1} \cdot 1+\alpha_{2} \cdot 2+\cdots+\alpha_{n} \cdot n=n\right\} .
$$

Let $|\alpha|=\sum_{i} \alpha_{i}$ and $g(\alpha)=\operatorname{gcd}\left\{k \mid \alpha_{k} \neq 0\right\}$. Then, there is a canonical isomorphism

$$
H^{i+2 n}\left(A \times \operatorname{Kum}^{n-1}(A)\right)=\bigoplus_{\alpha \in P(n)}\left(H^{i+2|\alpha|}\left(A^{(\alpha)}\right)\right)^{\oplus g(\alpha)^{4}}
$$

where $A^{(a)}=\operatorname{Sym}^{a}(A)$ for a positive integer a and $A^{(\alpha)}=A^{\left(\alpha_{1}\right)} \times \cdots \times A^{\left(\alpha_{n}\right)}$ for $\alpha=\left(\alpha_{1}, \cdots, \alpha_{n}\right)$ (we regard $A^{(0)}=\operatorname{Sym}^{0}(A)$ as a one point set). 
Let us consider $\mathscr{A} \times{ }_{\Delta^{*}} \operatorname{Kum}^{n}\left(\mathscr{A} / \Delta^{*}\right) \rightarrow \Delta$, which is in fact a degree $(n+1)^{4}$ étale cover of Hilb ${ }^{n+1}\left(\mathscr{A} / \Delta^{*}\right)$, and the associated monodromy operator $\tilde{T}_{m}$ on $H^{m}\left(\mathscr{A}_{t} \times \operatorname{Kum}^{n}\left(\mathscr{A}_{t}\right)\right)$.

Example 3.5. Consider the case where $n=2$, i.e., the monodromies on the cohomologies $H^{m}\left(\operatorname{Kum}^{2}\left(\mathscr{A}_{t}\right)\right)$ associated to $\pi: \operatorname{Kum}^{2}\left(\mathscr{A} / \Delta^{*}\right) \rightarrow \Delta^{*}$. We shall write $A=\mathscr{A}_{t}$ below for simplicity. By the Göttsche-Soergel formula (Theorem 3.4), we have

$$
H^{4}\left(A \times \operatorname{Kum}^{2}(A)\right)=H^{0}(A)^{\oplus 3^{4}} \oplus H^{2}(A \times A) \oplus H^{4}\left(A^{(3)}\right)
$$

and one can easily check

$$
\begin{aligned}
H^{2}(A \times A) & \cong H^{2}(A) \oplus 2 \oplus\left(H^{1}(A) \otimes H^{1}(A)\right), \\
H^{4}\left(A^{(3)}\right) & \cong H^{4}(A) \oplus\left(H^{3}(A) \otimes H^{1}(A)\right) \oplus \operatorname{Sym}^{2} H^{2}(A) \oplus\left(H^{2}(A) \otimes \wedge^{2} H^{1}(A)\right) .
\end{aligned}
$$

In particular, $\tilde{T}_{m}$ is unipotent. Take $\tilde{N}_{m}=\log \tilde{T}_{m}$. Using Lemma 2.4 and (3), we know that $\operatorname{nilp}\left(\tilde{N}_{4}\right)=2 l$, where $l=\operatorname{nilp}\left(\bar{N}_{2}\right) \geqslant 1$. On the other hand, the Künneth formula

$$
\begin{aligned}
& H^{4}\left(A \times \operatorname{Kum}^{2}(A)\right) \cong H^{4}\left(\operatorname{Kum}^{2}(A)\right) \oplus\left(H^{3}\left(\operatorname{Kum}^{2}(A)\right) \otimes H^{1}(A)\right) \\
& \oplus\left(H^{2}\left(\operatorname{Kum}^{2}(A)\right) \otimes H^{2}(A)\right) \oplus H^{4}(A)
\end{aligned}
$$

infers that $T_{m}$ is also unipotent and

$$
\operatorname{nilp}\left(\tilde{N}_{4}\right)=\max \left\{\operatorname{nilp}\left(N_{4}\right), \operatorname{nilp}\left(N_{3}\right)+1,2 l\right\}
$$

if $l>0 \Leftrightarrow \operatorname{nilp}\left(\bar{N}_{1}\right)=1$. Therefore

$$
2 l=\operatorname{nilp}\left(\tilde{N}_{4}\right) \geqslant \operatorname{nilp}\left(N_{4}\right) \geqslant 2 l
$$

where the last inequality is due to Lemma 3.3 Note that this holds also for $l=\operatorname{rank} \bar{N}_{1}=0$. In summary, we have

$$
\operatorname{nilp}\left(N_{4}\right)=2 \cdot \operatorname{nilp}\left(N_{2}\right) \text {. }
$$

Generalizing this method of calculation, we can show the following.

Theorem 3.6. Take $n \geqslant 2$ and $2<k \leqslant n$. Let $T_{m}$ be the monodromy operator on $H^{m}\left(\operatorname{Kum}^{n}\left(\mathscr{A}_{t}\right), \mathbb{C}\right)$ associated with the family $\pi: \operatorname{Kum}^{n}(\mathscr{A}) \rightarrow \Delta^{*}$ in Definition 3.1 Then, $T_{m}$ is also unipotent and

$$
\operatorname{nilp}\left(N_{2 k}\right)=k l, \quad \operatorname{nilp}\left(N_{2 k-1}\right) \leqslant k l-1 .
$$

for $N_{m}=\log T_{m}$. In particular $\operatorname{nilp}\left(N_{2 k}\right) \in\{0, k, 2 k\}$.

We prove this theorem via several steps. We keep the notation $A=\mathscr{A}_{t}$. 
Lemma 3.7. Let a be a positive integer and $N(m, a)$ the logarithm of the induced monodromy operator on $H^{m}\left(A^{(a)}\right)$. Then,

$$
\begin{aligned}
\operatorname{nilp}(N(2 M, a)) & = \begin{cases}M l & (M \leqslant a) \\
(2 a-M) l & (a<M \leqslant 2 a), \\
0 & (M>2 a)\end{cases} \\
\operatorname{nilp}(N(2 M+1, a)) & = \begin{cases}M l+1 & (M \leqslant a) \\
(2 a-M-1) l+1 & (a<M \leqslant 2 a) . \\
0 & (M>2 a)\end{cases}
\end{aligned}
$$

Proof. By the Poincaré duality, we have only to consider the case $M \leqslant a$. Let $\mu=\left(\mu_{1}, \cdots, \mu_{4}\right)$ be the partition of $m=2 M$ or $2 M+1$

$$
m=\mu_{1} \cdot 1+\mu_{2} \cdot 2+\mu_{3} \cdot 3+\mu_{4} \cdot 4
$$

under $|\mu|=\mu_{1}+\mu_{2}+\mu_{3}+\mu_{4} \leqslant a$. Then we have

$$
H^{m}\left(A^{(a)}\right) \cong \bigoplus_{\mu} H^{(\mu)}\left(A^{(a)}\right)
$$

where

$$
H^{(\mu)}\left(A^{(a)}\right) \cong \wedge^{\mu_{1}} H^{1}(A) \otimes \operatorname{Sym}^{\mu_{2}} H^{2}(A) \otimes \wedge^{\mu_{3}} H^{3}(A) \otimes \operatorname{Sym}^{\mu_{4}} H^{4}(A) .
$$

Let $N((\mu), a)$ be the logarithm of the induced monodromy operator on $H^{(\mu)}\left(A^{(a)}\right)$, then $\operatorname{nilp}(N(m, a))=\max _{\mu} \operatorname{nilp}(N((\mu), a))$. One can easily see that nilp $N((\mu), a)$ attains the maximum at

$$
\begin{aligned}
& \mu=(0, M, 0,0) \quad(m=2 M), \\
& \mu=(1, M, 0,0) \quad(m=2 M+1),
\end{aligned}
$$

and the corresponding maximum values are $M l$ and $M l+1$, respectively. Q.E.D.

Lemma 3.8. Notation as above. $\operatorname{nilp}\left(\tilde{N}_{2 k}\right)=k l$.

Proof. By the Göttsche-Soergel formula,

$$
H^{2 k}\left(A \times \operatorname{Kum}^{n}(A)\right)=\bigoplus_{\alpha \in P(n+1)}\left(H^{m_{k}(\alpha)}\left(A^{(\alpha)}\right)\right)^{\oplus g(\alpha)^{4}}
$$

where $m_{k}(\alpha)=2|\alpha|-2(n-k+1)$. Let $\tilde{N}_{2 k}(\alpha)$ be the logarithm of the induced monodromy on $H^{m_{k}(\alpha)}\left(A^{(\alpha)}\right)$ and $v(\alpha)=\#\left\{i \mid \alpha_{i} \neq 0\right\}$. Then, by the Künneth formula and Lemma 3.7, we have

$$
\begin{array}{r}
\operatorname{nilp}\left(\tilde{N}_{2 k}(\alpha)\right)=\max \left\{\frac{m_{k}(\alpha)}{2} \cdot l,\left(\frac{m_{k}(\alpha)}{2}-1\right) \cdot l+2,\right. \\
\left.\quad \cdots,\left(\frac{m_{k}(\alpha)}{2}-v(\alpha)\right) \cdot l+2 v(\alpha)\right\} .
\end{array}
$$


As we have an obvious inequality $|\alpha|+v(\alpha) \leqslant n+1$, which is immediate from $\alpha \in P(n+1)$, we have

$$
\left(\frac{m_{k}(\alpha)}{2}-v(\alpha)\right) \cdot l+2 v(\alpha)=(|\alpha|-n+k-v(\alpha)-1) \cdot l+2 v(\alpha) \leqslant k l
$$

even if $l=1$. Therefore, we get

$$
\operatorname{nilp}\left(\tilde{N}_{2 k}\right)=\max \left\{\operatorname{nilp}\left(\tilde{N}_{2 k}(\alpha)\right) \mid \alpha \in P(n+1)\right\} \leqslant k l .
$$

But nilp $\left(\tilde{N}_{2 k}(\alpha)\right)$ attains the maximum $k l$ when $\alpha=(n+1,0, \cdots)$. This completes the proof of the lemma.

Q.E.D.

Proof of Theorem 3.6 By the Künneth formula

$$
H^{2 k}\left(A \times \operatorname{Kum}^{n}(A)\right) \cong \bigoplus_{i=0}^{4} H^{i}(A) \otimes H^{2 k-i}\left(\operatorname{Kum}^{n}(A)\right),
$$

we have

$$
\begin{aligned}
\operatorname{nilp}\left(\tilde{N}_{2 k}\right)=\max \left\{\operatorname{nilp}\left(N_{2 k}\right)\right. & , \operatorname{nilp}\left(N_{2 k-1}\right)+1, \\
& \left.\operatorname{nilp}\left(N_{2 k-2}\right)+l, \operatorname{nilp}\left(N_{2 k-3}\right)+1, \operatorname{nilp}\left(N_{2 k-4}\right)\right\}
\end{aligned}
$$

In particular, $T_{m}$ is unipotent and

$$
\operatorname{nilp}\left(N_{2 k}\right) \leqslant k l \quad \operatorname{nilp}\left(N_{2 k-1}\right) \leqslant k l-1
$$

by Lemma 3.8. On the other hand, we have nilp $\left(N_{2 k}\right) \geqslant k l$ by Lemma 3.3, so this proves the theorem.

Q.E.D.

Remark 3.9. We should note that the proof of the equality (5) is limited to the case of the family of generalized Kummer varieties arising from a degeneration of abelian surfaces, unlike the case of Hilbert type (cf. Theorem 2.5]).

\section{GOOD DEGENERATION OF A COMPACT SYMPLECTIC KÄHLER MANIFOLD}

From what we have seen in the previous sections, we can easily pose the following question: Do the monodromy operators on the cohomologies associated with the degeneration of an irreducible symplectic manifold have some special property? To study the question in a somewhat general situation, it is certainly one way to consider the relation between the monodromies and the geometry of the singular fiber. Along this direction, we have the powerful theory of the limit mixed Hodge structure in the case of semi-stable degenerations.

Definition 4.1. Let $M$ be a complex manifold. A divisor $D$ on $M$ is a simple normal crossing divisor (SNC in short) if $D=\sum D_{i}$ is reduced, every irreducible component $D_{i}$ is smooth, and for any point $p \in D$, the local equation of $D$ in $M$ is given by $x_{1} \cdots x_{r}=0$. A degeneration $\pi: \mathscr{X} \rightarrow \Delta$ is semi-stable if the total space $\mathscr{X}$ is smooth and $X=\mathscr{X}_{0}=\pi^{-1}(0)$ is a SNC divisor as scheme theoretic fiber. 
By the semi-stable reduction theorem, a degenerating family is always birational to a semi-stable one after taking some cyclic base change. It is also known that the monodromies $T_{m}$ on $H^{m}\left(\mathscr{X}_{t}, \mathbb{C}\right)$ is unipotent if the degeneration $\pi: \mathscr{X} \rightarrow \Delta$ is semi-stable. In this sense, we can consider a semi-stable degeneration as a geometric counterpart of the concept of "unipotent monodromy".

But one should note that there are many semi-stable models for a given degenerating family since one can operate birational modifications keeping the family semi-stable. To carry out some geometric arguments on the singular fiber, it is desirable to have a kind of "minimality" of the family. As that kind of thing, we propose the following definition of a good degeneration of a compact symplectic Kähler manifold.

Definition 4.2. A good degeneration of compact symplectic Kähler manifold is a degeneration $\pi: \mathscr{X} \rightarrow \Delta$ of relative dimension $2 n$ satisfying

(i) $\pi$ is semi-stable.

(ii) There exists a relative logarithmic 2-form $\sigma_{\pi} \in H^{0}\left(\mathscr{X}, \Omega_{\mathscr{X} / \Delta}^{2}(\log X)\right)$ such that $\wedge^{n} \sigma_{\pi} \in H^{0}\left(\mathscr{X}, K_{\mathscr{X} / \Delta}\right)$ is nowhere vanishing (see, for example, [St] for the definition of the logarithmic differential forms).

Note that the condition (ii) implies that $K_{\mathscr{X} / \Delta}$ is trivial. In particular the definition above agrees with the definition of good degeneration of K3 surface, socalled Kulikov model, by Kulikov-Persson-Pinkham $[\mathrm{Ku}, \mathrm{P}-\mathrm{P}]$ if $n=1$. This definition can be seen as an analogy of a smooth minimal model in the case of degeneration.

Let us construct an example of a good degeneration of an irreducible symplectic manifold using the degenerating family of the Hilbert schemes on K3 surfaces in $\S 2$.

Theorem 4.3. Let $p: \mathscr{S} \rightarrow \Delta$ be a type II degeneration of $K 3$ surface, i.e. $p$ is a good degeneration of $K 3$ surface with the singular fiber $\mathscr{S}_{0}=S_{0} \cup S_{1} \cup \cdots \cup$ $S_{k-1} \cup S_{k}$ where $S_{0}$ and $S_{k}$ are rational surfaces, $S_{i}(0<i<k)$ are elliptic ruled surfaces and $S_{i}$ meets only $S_{i \pm 1}$ in smooth elliptic curves $C_{i}=S_{i} \cap S_{i+1}(i=$ $0, \cdots k-1)$. Consider the relative Hilbert scheme $\rho: \mathscr{Y}=\operatorname{Hilb}^{2}(\mathscr{S} / \Delta) \rightarrow \Delta$ of relative sub-schemes of length 2. Then there exists a projective birational morphism $\mu: \mathscr{X} \rightarrow \mathscr{Y}$ such that

$$
\pi=\rho \circ \mu: \mathscr{X} \rightarrow \Delta
$$

is a good degeneration of compact symplectic Kähler manifold.

In the proof of the proposition given below, it is essential the condition that the length of sub-schemes in question is 2 . It is natural to ask either the same conclusion holds for the larger length sub-schemes. To answer this question, it is likely that a more intrinsic interpretation of the resolved space $\mathscr{X}$ is needed. 
Proof. The family $p: \mathscr{S} \rightarrow \Delta$ induces the morphism

$$
f: \operatorname{Hilb}^{2}(\mathscr{S}) \rightarrow \operatorname{Sym}^{2}(\mathscr{S}) \rightarrow \operatorname{Sym}^{2}(\Delta) .
$$

We can consider $\operatorname{Hilb}^{2}(\mathscr{S} / \Delta)$ as a closed sub-scheme of $\operatorname{Hilb}^{2}(\mathscr{S})$. Moreover if we define $d: \Delta \rightarrow \operatorname{Sym}^{2}(\Delta)$ by $z \mapsto 2[z]$, then we have the commutative diagram

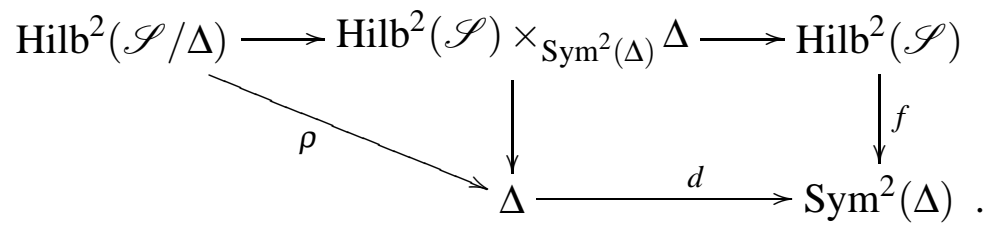

A singular sub-scheme of length 2 is given as the base point plus tangent direction at that point and any sub-scheme of length 2 is given as a limit of smooth sub-schemes (i.e., sub-schemes consisting of different 2 points). Therefore

$$
\operatorname{Hilb}^{2}(\mathscr{S})=B l_{D}(\mathscr{S} \times \mathscr{S}) / \mathfrak{S}_{2}
$$

where $D$ is the diagonal, $B l_{D}$ stands for the blowing up along $D$ and $\mathfrak{S}_{2}$ acts as the permutation of components. Moreover $\operatorname{Hilb}^{2}(\mathscr{S} / \Delta)$ is the closure of the open subset of $\operatorname{Hilb}^{2}(\mathscr{S}) \times_{\operatorname{Sym}^{2}(\Delta)} \Delta$ consisting of points corresponding to the smooth sub-schemes. Thus we have the following commutative diagram

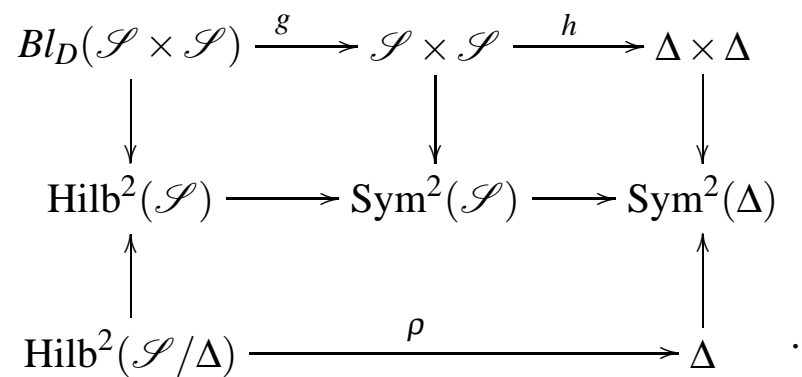

Let $Z$ be the strict transform on $B l_{D}(\mathscr{S} \times \mathscr{S})$ of the inverse image by $h$ of the diagonal of $\Delta \times \Delta$. Then we have the commutative diagram

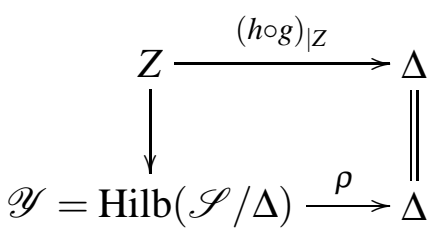

where the first vertical arrow is the quotient map by the action of $\mathfrak{S}_{2}$.

Now, since $\mathscr{S}$ is a type II degeneration of K3 surfaces, components of $\mathscr{Y}_{0}=$ $\rho^{-1}(0)$ consists of $Y_{i j}(0 \leqslant i \leqslant j \leqslant k)$ where $Y_{i i}=\operatorname{Hilb}^{2}\left(S_{i}\right)$ and $Y_{i j}$ has selfintersection and its normalization is $S_{i} \times S_{j}$. The configuration of these components is as follows. 


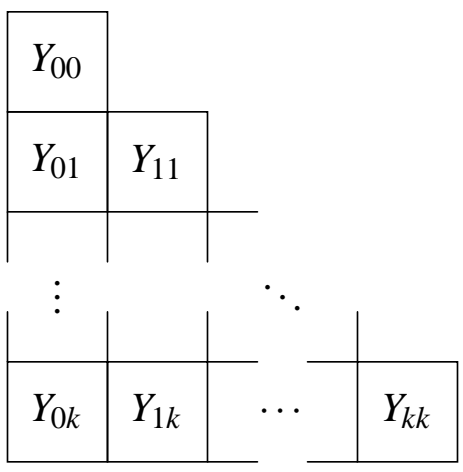

Now we define $\mu: \mathscr{X} \rightarrow \mathscr{Y}$ as the blowing up along $C=\bigcup Y_{2 s, 2 k}$.

$$
0 \leqslant 2 s \leqslant 2 t \leqslant k
$$

We want to show $\pi: \mathscr{X} \rightarrow \Delta$ is a semi-stable degeneration. Noting that $\rho$ is smooth over $\Delta^{*}=\Delta-\{0\}$, we have only to look at the singular fiber. We have to get local description of singularities along the intersection of these components. Away from the diagonal, $\operatorname{Hilb}^{2}(\mathscr{S})$ is locally the direct product of open neighborhoods of $\mathscr{S}$ and $f$ is given by

$$
\left(z_{1}, \ldots, z_{6}\right) \mapsto\left(z_{1} z_{2}, z_{4} z_{5}\right)
$$

so that total space of $\rho$ is defined by $z_{1} z_{2}-z_{4} z_{5}=0$ and the central fiber of $\rho$ is defined by $z_{1} z_{2}=z_{4} z_{5}=0$. As the center $C$ of $\mu$ is defined by, say, $z_{1}=z_{4}=0$, the defining equation of $\mathscr{X}$ is given by $z_{1}^{\prime} z_{2}^{\prime}-z_{5}^{\prime}=0$ and $\pi=\rho \circ \mu$ is described by

$$
\left(z_{1}^{\prime}, \ldots, z_{6}^{\prime}\right) \mapsto z_{5}^{\prime}
$$

This shows $\mathscr{X}$ is smooth and $X=\pi^{-1}(0)$ is normal crossing divisor in this coordinate neighborhood.

Next we consider local description of $[Z] \in \rho^{-1}(0)$ with $\operatorname{supp} Z=\{q\}, q \in \mathscr{S}_{0}$. Take a local coordinate $\left(z_{1}, \ldots, z_{6}\right)$ of $(q, q) \in \mathscr{S} \times \mathscr{S}$. The defining equation of diagonal $D$ is $z_{1}-z_{4}=z_{2}-z_{5}=z_{3}-z_{6}=0$. Put

$$
\begin{aligned}
& x_{1}=z_{1}+z_{4}, \quad x_{3}=z_{2}+z_{5}, \quad x_{5}=z_{3}+z_{6} \text {, } \\
& x_{2}=z_{1}-z_{4}, \quad x_{4}=z_{2}-z_{5}, \quad x_{6}=z_{3}-z_{6} \text {. }
\end{aligned}
$$

The map $h$ is given by

$$
\left(x_{1}, \ldots, x_{6}\right) \mapsto\left(\left(x_{1}+x_{2}\right) \cdot\left(x_{3}+x_{4}\right),\left(x_{1}-x_{2}\right) \cdot\left(x_{3}-x_{4}\right)\right) .
$$

The defining ideal of $D$ is $\left(x_{2}, x_{4}, x_{6}\right)$ and a piece of the blowing up $g$ is locally described by

$$
\begin{array}{rc}
x_{1}=y_{1}, \quad x_{2}=y_{2} y_{6}, & x_{3}=y_{3}, \\
x_{4}=y_{4} y_{6}, \quad x_{5}=y_{5} & x_{6}=y_{6} .
\end{array}
$$

and $g \circ h$ is given by

$$
\left(y_{1}, \ldots, y_{6}\right) \mapsto\left(\left(y_{1}+y_{2} y_{6}\right)\left(y_{3}+y_{4} y_{6}\right),\left(y_{1}-y_{2} y_{6}\right)\left(y_{3}-y_{4} y_{6}\right)\right) .
$$


Restricting on $Z$, i.e., imposing the condition

$$
y_{2} y_{3}+y_{1} y_{4}=0
$$

$g \circ h$ is given by

$$
\left(y_{1}, \ldots, y_{6}\right) \mapsto y_{1} y_{3}+y_{2} y_{4} y_{6}^{2}
$$

Noting that the action of $\mathfrak{S}_{2}$ is $\operatorname{diag}(1,1,1,1,1,-1), \rho$ is described by

$$
\left(w_{1}, \ldots, w_{6}\right) \mapsto w_{1} w_{3}+w_{2} w_{4} w_{6}
$$

under the condition $w_{2} w_{3}+w_{1} w_{4}=0$. In this coordinate, the equation of the center $C$ of $\mu$ is, say, $w_{1}=w_{2}=0$. Therefore the equation of $\mathscr{X}$ is given by $w_{3}^{\prime}+w_{1}^{\prime} w_{4}^{\prime}=0$ and $\pi=\rho \circ \mu$ is described by

$$
\left(w_{1}^{\prime}, \ldots, w_{6}^{\prime}\right) \mapsto w_{1}^{\prime} w_{2}^{\prime} w_{3}^{\prime}+w_{2}^{\prime} w_{4}^{\prime} w_{6}^{\prime} .
$$

The fiber $\pi^{-1}(t)$ is $w_{2}^{\prime} w_{4}^{\prime}\left(w_{6}^{\prime}-w_{1}^{\prime 2}\right)=t$ in the coordinate $\left(w_{1}^{\prime}, w_{2}^{\prime}, w_{4}^{\prime}, w_{5}^{\prime}, w_{6}^{\prime}\right)$. These calculations show that $\pi$ is semi-stable also in this coordinate neighborhood. We remark that the configuration of components of $\mathscr{X}_{0}=\pi^{-1}(0)$ is as follows.

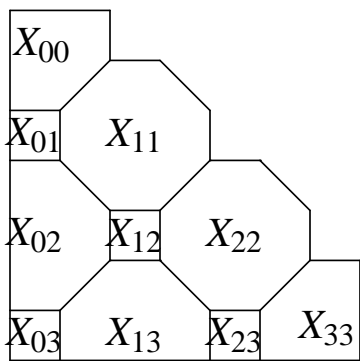

This is the case where $k=3$.

It remains to show that there exists a relative logarithmic symplectic form $\sigma_{\pi} \in$ $H^{0}\left(\mathscr{X}, \Omega_{\mathscr{X} / \Delta}^{2}(\log X)\right)$. The original family $p: \mathscr{S} \rightarrow \Delta$ have nowhere vanishing relative 2 -form $\omega$. We claim that the relative 2 -form $\widetilde{\omega}=\operatorname{pr}_{1}^{*} \omega+\operatorname{pr}_{2}^{*} \omega$ on $\mathscr{S} \times$ $\mathscr{S} \rightarrow \Delta \times \Delta$ induces $\sigma_{\pi}$. The restriction of $\widetilde{\omega}$ to the inverse image by $h$ of the diagonal of $\Delta \times \Delta$ is invariant under the action of $\mathfrak{S}_{2}$, therefore $\left(g^{*} \widetilde{\omega}\right)_{\mid Z}$ descends to a 2 -form $\varphi$ on $\operatorname{Hilb}^{2}(\mathscr{S} / \Delta)$. Let $\sigma_{\pi}=\mu^{*} \varphi$. As $\varphi$ is non-degenerate outside the critical points of $\pi$ by the argument same as in [Be], we know that $K_{\mathscr{Y}}=0$. By the theory of limiting Hodge structure induced by a semi-stable degeneration [St], the direct image sheaf $\pi_{*} \Omega_{\mathscr{X} / \Delta}^{2}(\log X)$ is locally free. Since $\mu: \mathscr{X} \rightarrow \mathscr{Y}$ is a small resolution, $K_{\mathscr{X}}$ is trivial. Therefore we see that $\sigma_{\pi}$ above defines everywhere non-degenerate section of the invertible sheaf $\pi_{*} \Omega_{\mathscr{X} / \Delta}^{2}(\log X)$. This shows that $\sigma_{\pi}$ satisfies the condition of Definition 4.2, (ii).

Q.E.D.

Remark 4.4. We may construct similar example from type III degenerations of $\mathrm{K} 3$ surfaces. But in these cases, the combinatorics of the components becomes more complicated and the choice of the center of a resolution should be subtle. 
We should also remark that our definition of good degeneration may be "too good" in general. In view of the situation of the complexity of the minimal models in higher dimensions, it is too optimistic to expect a good degeneration model for a given degeneration of irreducible symplectic Kähler manifolds. For example, Kulikov-Persson-Pinkham model can be seen as a relatively minimal 3 -fold model over the base and their construction of the good model is already quite complicated.

\section{MONODROMY OF A GOOD DEGENERATION OF AN IRREDUCIBLE SYMPLECTIC MANIFOLD}

Now we consider the behavior of the monodromy operators on the cohomologies associated to a good degeneration of compact irreducible symplectic Kähler manifolds.

Inspired by Theorem 2.5 and 3.6 we propose the following conjecture.

Conjecture 5.1. Let $\pi: \mathscr{X} \rightarrow \Delta$ be a degeneration of an irreducible symplectic $2 n$-fold, $T_{m}$ the associated monodromy operator on $H^{m}\left(\mathscr{X}_{t}, \mathbb{C}\right)$. Assume $T_{m}$ is unipotent and put $N_{m}=\log T_{m}$. Then $\operatorname{nilp}\left(N_{2 k}\right)=k \cdot \operatorname{nilp}\left(N_{k}\right)$ for $k \leqslant n$.

We can also consider a weak version of this conjecture.

Conjecture 5.2. Under the assumption and notation as in Conjecture 5.1] we have $\operatorname{nilp}\left(N_{2 k}\right) \in\{0, k, 2 k\}$.

Of course, Conjecture 5.1 implies Conjecture 5.2. We have already seen that these conjectures are ture if

(i) The general fiber $\mathscr{X}_{t}$ is of Hilbert type (Theorem 2.5).

(ii) $\pi$ is the family of generalized Kummer manifolds $\pi: \operatorname{Kum}^{2}(\mathscr{A} / \Delta) \rightarrow \Delta$ as in Definition 3.1 (Theorem 3.6).

These conjectures could be too naive for the higher dimensions. The author suspect that the conjectures may be true at least for lower dimensions, for example $2 n=4$ and 6 .

Under the assumption of the existence of a good degeneration model, we can easily prove the following theorem, which is a partial answer to Conjecture 5.2

Theorem 5.3. Let $\pi: \mathscr{X} \rightarrow \Delta$ be a good degeneration of irreducible symplectic $2 n$-folds. Let $H_{t}^{m}=H^{m}\left(\mathscr{X}_{t}, \mathbb{C}\right)(t \neq 0), T_{m}$ be the monodromy operator on $H_{t}^{m}$ associated to the family $\pi$, and $N_{m}=\log T_{m}$. Take $k \leqslant n$ and assume $N_{2 k}^{k}=0$, then $N_{2 k}=0$, In other words, $\operatorname{nilp}\left(N_{2 k}\right) \in\{0, k, k+1, \cdots, 2 k\}$.

Proof. Let $H^{m}=H^{m}(X, \mathbb{C})$ be the cohomology of the singular fiber $X=\mathscr{X}_{0}$. Consider the Clemens-Schmid exact sequence (a convenient reference is Morrison's lecture note, [Topics], Chapter VI):

$$
F^{m} \mathrm{Gr}_{m}^{W} H^{m} \longrightarrow F^{m} \mathrm{Gr}_{m}^{W} H_{t}^{m} \stackrel{N_{m}}{\longrightarrow} F^{m-1} \mathrm{Gr}_{m-2}^{W} H_{t}^{m}
$$


The last term must be zero because $\mathrm{Gr}_{m-2}^{W} H_{t}^{m}$ is a pure Hodge structure of weight $(m-2)$.

Let $X^{[p]}=\bigsqcup X_{i_{0} \cdots i_{p}}$ where $X_{i_{0} \cdots i_{p}}=X_{i_{0}} \cap \cdots \cap X_{i_{p}}$ the $p$-fold intersection of the components of the singular fiber $X=\sum X_{i}$. Since the weight spectral sequence

$$
{ }_{W} E_{1}^{p q}=H^{q}\left(X^{[p]}, \mathbb{C}\right) \Rightarrow E^{p+q}=H^{q+p}(X, \mathbb{C})
$$

is $E_{2}$-degenerate and $d_{1}^{p q}$ is compatible with the Hodge filtration, we have

$$
F^{m} \mathrm{Gr}_{m}^{W} H^{m}=F^{m} \operatorname{Ker}\left(H^{m}\left(X^{[0]}\right) \stackrel{d_{1}^{0, m}}{\longrightarrow} H^{m}\left(X^{[1]}\right)\right) .
$$

and $h^{m, 0}\left(X^{[0]}\right) \geqslant \operatorname{dim} F^{m} \operatorname{Gr}_{m}^{W} H_{t}^{m}$. On the limit Hodge structure $H_{t}^{m}$, we have

$$
\begin{aligned}
h^{m, 0}\left(\mathscr{X}_{t}\right) & =\operatorname{dim} F^{m} H_{t}^{m} \\
& =\operatorname{dim} F^{m} \mathrm{Gr}_{m}^{W} H_{t}^{m}+\operatorname{dim} F^{m} \mathrm{Gr}_{m+1}^{W} H_{t}^{m}+\cdots+\operatorname{dim} F^{m} \operatorname{Gr}_{2 m}^{W} H_{t}^{m} .
\end{aligned}
$$

As $\mathscr{X}_{t}$ is an irreducible symplectic manifold, we have $h^{2,0}\left(\mathscr{X}_{t}\right)=1$. The condition $N_{2 k}^{k}=0$ implies $N_{2}=0$ by Lemma 3.3, hence $\mathrm{Gr}_{3}^{W} H_{t}^{2}=\mathrm{Gr}_{4}^{W} H_{t}^{2}=$ 0 . Therefore, $h^{2,0}\left(X^{[0]}\right) \geqslant \operatorname{dim} F^{2} \operatorname{Gr}_{2}^{W} H_{t}^{2}=h^{2,0}\left(\mathscr{X}_{t}\right)=1$, which means that there exists a component $X_{0} \subset X$ such that we have a non-zero section $\eta \in$ $H^{0}\left(X_{0}, \Omega_{X_{0}}^{2}\right)$. Thus, we have a section $0 \neq \eta^{\wedge n} \in H^{0}\left(X_{0}, K_{X_{0}}\right)$, in other words, $K_{X_{0}}$ is effective.

On the other hand, since $\pi$ is a good degeneration of symplectic manifold, there exists a non-degenerate logarithmic 2-form $\sigma \in H^{0}\left(X, \Omega_{X / \mathbb{C}}^{2}(\log )\right)$. Let $D_{0}=X_{0} \cap\left(\bigcup_{i \neq 0} X_{i}\right)$. Then $\sigma_{\mid X_{0}} \in H^{0}\left(X_{0}, \Omega_{X_{0}}^{2}\left(\log D_{0}\right)\right)$ is a non-degenerate section, i.e., $\sigma_{\mid X_{0}}^{\wedge n} \in H^{0}\left(X_{0}, K_{X_{0}}+D_{0}\right)$ is nowhere vanishing. This implies, $K_{X_{0}} \sim$ $-D_{0}$. If $N_{2 k} \neq 0$, then $X$ has more than one component and $D_{0}$ is effective. This contradicts to the fact that $K_{X_{0}}$ is effective.

Q.E.D.

Remark 5.4. The proof shows in fact that $N_{2}=0 \Leftrightarrow N_{2 k}=0$. Therefore, this theorem is also a partial answer to Conjecture 5.1

Corollary 5.5. Notation as above. For a good degeneration of irreducible symplectic 2 -folds with non-trivial monodromy on the middle cohomology, we have $X^{[p]} \neq \emptyset$ for $p \leqslant n$. In other words, for the dual graph $\Gamma$ of the configuration of the irreducible components of the singular fiber $X$, the dimension of the topological realization $|\Gamma|$ is at least $n$.

Proof. According to the weight spectral sequence (8), we have

$$
\operatorname{Gr}_{q}^{W} H^{2 n} \cong \frac{\operatorname{Ker}\left(H^{q}\left(X^{[2 n-q]}\right) \rightarrow H^{q}\left(X^{[2 n-q+1]}\right)\right)}{\operatorname{Im}\left(H^{q}\left(X^{[2 n-q-1]}\right) \rightarrow H^{q}\left(X^{[2 n-q]}\right)\right)}
$$

By the Clemens-Schmid exact sequence, we also have

$$
\mathrm{Gr}_{q}^{W} H^{2 n} \cong \mathrm{Gr}_{q}^{W} \operatorname{Ker}\left(H_{t}^{2 n} \stackrel{N}{\longrightarrow} H_{t}^{2 n}\right)
$$


for $q<2 n$. If we have $X^{[n]}=\emptyset$, (9) implies $\operatorname{Gr}_{q}^{W} H^{2 n}=0$ for $q \leqslant n$, therefore

$$
\mathrm{Gr}_{q}^{W} H_{t}^{2 n}=\bigoplus_{i=0}^{\lfloor q / 2\rfloor} \mathrm{Gr}_{q-2 i}^{W} \operatorname{Ker}\left(H_{t}^{2 n} \stackrel{N}{\longrightarrow} H_{t}^{2 n}\right)=0
$$

for $q \leqslant n$, i.e., $N_{2 n}^{n}=0$. By Theorem 5.3 , we get $N_{2 n}=0$, which is a contradiction.

Q.E.D.

This corollary means in particular that there is no chain degeneration nor cycle degeneration of irreducible symplectic manifold, i.e. no good degeneration such that the dual graph of the singular fiber is as following:
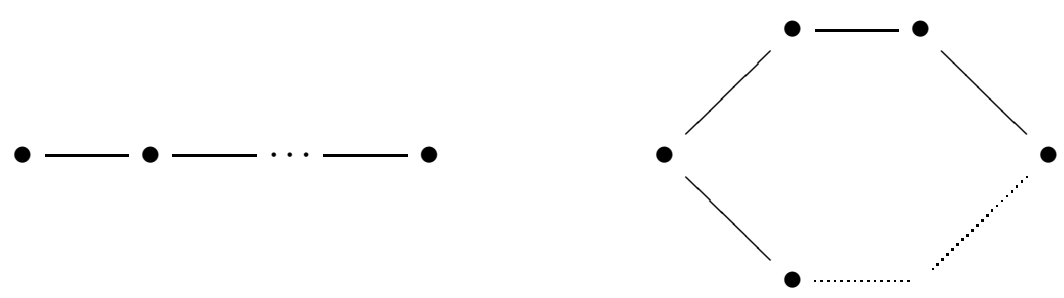

It is remarkable to compare this to the fact that one can generically expect chain degenerations for Calabi-Yau manifolds and cyclic degenerations for abelian varieties. We can regard the situation in the example of Theorem 4.3 is the least degenerate case of the degeneration of symplectic manifolds. We should also note that we can expect some special property of the period map on the middle cohomology of irreducible symplectic manifolds.

To end the article, we discuss about the relationship between the conjecture for more highly degenerate case, i.e., the case where nilp $\left(N_{2 k}\right)>k$ and the singular fiber of a good degeneration of irreducible symplectic manifolds.

Proposition 5.6. Notation as in Theorem 5.3 Assume that $N_{2 n}^{2 n}=0$. Then we have $N_{2 n}^{2 n-1}=0$ if and only if for every elliptic curve $C$ contained in $2 n$-tuple intersection $X^{[2 n-1]}$, there exists a surface component $S \subset X^{[2 n-2]}$ such that $S \supset C$ and the irregularity $q(S)>0$.

If $n=2$, the proposition asserts the conjecture 5.2. under the artificial condition of the intersections.

Proof. The conclusion $N_{2 n}^{2 n-1}=0$ is equivalent to $\mathrm{Gr}_{1}^{W} H_{t}^{2 n}=0$ under the condition $N_{2 n}^{2 n}=0$. We have

$$
\mathrm{Gr}_{1}^{W} H_{t}^{2 n}=\mathrm{Gr}_{1}^{W}(\operatorname{Ker} N) \cong \mathrm{Gr}_{1}^{W} H^{2 n}
$$

where the last isomorphism is due to Clemens-Schmid exact sequence. Again by the weight spectral sequence on an SNC variety (8), we have

$$
\mathrm{Gr}_{1}^{W} H^{2 n} \cong \frac{H^{1}\left(X^{[2 n-1]}\right)}{\operatorname{Im}\left(H^{1}\left(X^{[2 n-2]}\right) \rightarrow H^{2}\left(X^{[2 n-1]}\right)\right)} .
$$


We want to show that the vanishing of this vector space is equivalent to the existence of a surface mentioned in the statement of the theorem.

Lemma 5.7. For a connected component $Z \subset X_{i_{0} \cdots i_{r}}$ in the $(r+1)$-tuple intersection of the components of the singular fiber $X$,

$$
K_{Z}=-\sum_{Z \cap X_{j} \neq \emptyset} X_{i_{0} \cdots i_{r}, j} \cap Z,
$$

In particular, the genus $g(C)$ of a $2 n$-tuple curve $C \subset X_{i_{0} \cdots i_{2 n-1}}$ satisfies

$$
2 g(C)-2=-(\# \text { of }(2 n+1) \text {-tuple points on } C) \leqslant 0 \text {. }
$$

This is just a recurrence of the adjunction formula, since $K_{\mathscr{X}}$ is trivial and $X=$ $\sum X_{i} \sim 0$. By this lemma, we have two possibilities for a $2 n$-tuple curve $C$ :

(1) $C \cong \mathbb{P}^{1}$ and we have exactly two $(2 n+1)$-tuple points on $C$.

(2) $C$ is an elliptic curve and we have no $(2 n+1)$-tuple point on $C$.

Since $H^{1}\left(X^{[2 n-1]}\right)=\bigoplus_{C \subset X^{[2 n-1]}} H^{1}(C)$, a rational curve component $C$ has no contribution to $\mathrm{Gr}_{1}^{W} H^{2 n}$ so that we have only to care the elliptic curve components (In fact, we know that there is no $(2 n+1)$-tuple point on the singular fiber under the condition $N_{2 n}^{2 n}=0$, according to the result of Lian-Todorov-Yau [LTY], therefore, no rational curve in $X^{[2 n-1]}$ ).

Let $S_{k} \subset X_{i_{0} \cdots \hat{i}_{k} \cdots i_{2 n-1}}$ be a $(2 n-1)$-tuple surface containing an elliptic curve component $C \subset X_{i_{0} \cdots i_{2 n-1}}$. Then by Lemma 5.7 the anti-canonical divisor $-K_{S_{k}}$ is effective so that $S_{k}$ must be a rational surface or a (non-rational) ruled surface.

If $S_{k}$ is rational, a morphism $H^{1}\left(S_{k}\right) \rightarrow H^{1}(C)$ is obviously 0-map. If $S_{k}$ is a ruled surface, $C$ must be a section or bi-section of the ruling of $S_{k}$ since $C$ is a component of an effective SNC member of $\left|-K_{S}\right|$. This implies that $S_{k} \rightarrow E$ must be elliptic ruled and the composition $C \hookrightarrow S_{k} \rightarrow E$ is surjective. Therefore a natural map $H^{1}\left(S_{k}\right) \rightarrow H^{1}(C)$ is an isomorphism so that the component $H^{1}(C) \subset$ $H^{1}\left(X^{[2 n-1]}\right)$ is in the image of $H^{1}\left(X^{[2 n-2]}\right) \rightarrow H^{2}\left(X^{[2 n-1]}\right)$.

If for every elliptic curve $C$ in $X^{[2 n-1]}$ there exists a surface component $S_{k} \subset$ $X^{[2 n-2]}$ containing $C$ with $q\left(S_{k}\right)>0$, then $\mathrm{Gr}_{1}^{W} H^{2 n}=0 \Leftrightarrow N_{2 n}^{2 n-1}=0$. On the other hand, If $N_{2 n}^{2 n-1}=0$, then for every elliptic curve in $X^{[2 n-1]}$, there must be an elliptic ruled surface $S_{k} \supset C$, which must have positive irregularity. Q.E.D.

We note that it is the only assumption used in the proof of the proposition that the total space of the degeneration has trivial canonical bundle. To obtain more substantial result along this line of argument, we have to know more or less about geometric structures of the singular fiber of a good degeneration of irreducible symplectic Kähler manifolds. For a good degeneration of an irreducible symplectic manifold, we expect two strong constraints: one for the geometry of the components of the singular fiber and the other for the combination of the singular fibers. 
A variety which can be a component of the singular fiber is formulated as follows:

Definition 5.8. Let $X$ be a compact Kähler manifold of dimension $2 n$ and $D$ be a SNC divisor on it. The pair $(X, D)$ is called a log symplectic pair if there exists a non-degenerate logarithmic 2-form $\sigma \in H^{0}\left(X, \Omega_{X}^{2}(\log D)\right)$.

A log symplectic pair is a $\log$ Calabi-Yau in the sense that $K_{X}+D \sim 0$. This implies that $\kappa(X)=-\infty$. The log symplectic pairs in dimension 2 are classified in $[\mathrm{Ku}]$. The classification was used to determine the geometry of the singular fiber of Kulikov model and we also used it in the proof of the proposition above.

Example 5.9. We touch on some examples (and non-examples) of higher dimensional log symplectic pairs.

(i) a product of a log symplectic pairs is obviously a log symplectic pair, so that we can produce an example of log symplectic pairs in any dimensions from log symplectic surfaces.

(ii) $\left(\mathbb{P}^{2 n}, D\right)$ where $D$ is a SNC divisor such that $D \in\left|-K_{\mathbb{P} 2 n}\right|$ is not a log symplectic pair because $H^{2}(X-D, \mathbb{C})=0$ and therefore $H^{0}\left(X, \Omega_{X}^{2}(\log D)\right)=0$.

(iii) Let $Z$ be a compact symplectic Kähler manifold and $X \rightarrow Z$ a projective smooth surface bundle whose fiber has Kodaira dimension $\kappa=-\infty$. Let $D$ be a SNC divisor such that every component of $D$ dominates $Z$ and the restriction of $D$ to the fiber is an anti-canonical divisor. Then $(X, D)$ is a $\log$ symplectic pair with $h^{0}\left(X, \Omega_{X}^{2}(\log D)\right)=h^{0}\left(Z, \Omega_{Z}^{2}\right)$. This is a slight generalization of (i).

(iv) Let $(S, C)$ be a $\log$ symplectic surface such that $S$ is rational and $C$ is an elliptic curve on it. Let $Y=\operatorname{Hilb}^{2}(S)$ and $D_{1}=\left\{[Z] \in \operatorname{Hilb}^{2}(S) \mid \operatorname{supp} Z \cap\right.$ $C \neq \emptyset\}, \mu: X \rightarrow Y$ be a blowing up at $C^{\prime}=\operatorname{Hilb}^{2}(C) \subset Y=\operatorname{Hilb}^{2}(S)$, and $D_{2}$ the exceptional divisor of $\mu$. Then $\left(X, D=D_{1}+D_{2}\right)$ is a log symplectic pair with $h^{0}\left(X, \Omega_{X}^{2}(\log D)\right)=1$. In fact, this example is obtained in the course of the proof of Theorem 4.3 as the component $X_{00}$ or $X_{k k}$. Starting from an elliptic ruled surface $S$ and its sections $C=C_{1}+C_{2}$, we obtain another example, which is nothing but $X_{11}, \cdots, X_{k-1, k-1}$ in Theorem 4.3 .

It does not seem very easy to produce "essentially" new example of log symplectic pairs in higher dimensions, for example a log symplectic pair $(X, D)$ of dimension $\geqslant 4$ with $h^{0}\left(X, \Omega_{X}^{2}(\log D)\right)=1$, as in the case of the construction of irreducible symplectic manifolds. This is also the strong constraint originated from the presence of a symplectic form. The combinatorial problem to patch up these log symplectic pair to a "degenerate symplectic variety" is global in nature so that it will be much harder.

\section{REFERENCES}

[Be] Beauville, A., Variétés Kähleriennes dont la première classe de Chern est nulle, J. Diff. Geom. 18 (1983), No.4, 775-782. 
[Bo] Bogomolov, F.A., On the cohomology ring of a simple hyper-Kähler manifold (on the results of Verbitsky), Geom. Funct. Anal. 6 (1996), No.4, 612-618.

[Fr] Friedman, R., A New Proof of the Global Torelli Theorem for K3 surfaces, Ann. Math. 120 (1984), No.2, 237-269.

[GS] Göttsche, L., Soergel, W., Perverse sheaves and the cohomology of Hilbert schemes of smooth algebraic surfaces, Math. Ann. 296 (1993), No.2, 235-245.

[Topics] Griffiths, P., ed. Topics in Transcendental algebraic geometry, Annals of Math. Studies, 106, Princeton University Press (1984)

[Huy1] Huybrechts, D., Compact hyper-Kähler manifolds: basic results, Invent. Math. 135 (1999), No.1, 63-113, Erratum, 152 (2003), No.2, 209-212.

[Huy2] Huybrechts, D., Compact hyperKähler manifolds, Calabi-Yau manifolds and related geometries (Nordfjordeid, 2001), 161-225, Springer-Verlag (2003)

[Ku] Kulikov, V., Degenerations of K3 end Enriques surfaces, Math. USSR Izv. 11 (1977), No.5, 957-989. English translation: Math. USSR-Izv. 11 (1977), No.5, 957-989.

[LTY] Lian, B.H., Todorov, A., Yau, S-T., Maximal unipotent monodromy for complete intersection CY manifolds, Amer. J. Math. 127 (2005), 1-50.

[OG1] O'Grady, K.G., Desingularized moduli spaces of shaves on a K3, J. Reine Angew. Math. 512 (1999), 49-117.

[OG2] O'Grady, K.G., A new six-dimensional irreducible symplectic variety, J. Alg. Geom. 12 (2003), No.3, 435-505.

[P-P] Persson, P., Pinkham, H., Degeneration of surfaces with trivial canonical bundle, Ann. Math. 113 (1981), 45-66.

[Sc] Schmid, W., Variation of Hodge structure: the singularities of the period mapping, Invent. Math. 22 (1973), 211-319

[St] Steenbrink, J., Limits of Hodge Structures, Invent. math. 31 (1976), 229-257.

Korea Institute for Advanced Study (KIAS), 207-43 Cheongnyangni 2-Dong, Dondaemun-Gu, SeOul 130-722, Korea

E-mail address: nagai@kias.re.kr 